\title{
LA INTELIGENCIA EN LA EMPRESA Y EL MARKETING DE AFILIACIÓN
}

\author{
Clide Rodríguez Vázquez ${ }^{1}$ \\ Magdalena Rodríguez Fernández ${ }^{2}$ \\ Universidad de A Coruña \\ crodriguezv@udc.es \\ mmrodriguez@udc.es
}

Material original autorizado para su primera publicación en la revista académica REDMARKA. Revista Digital de Marketing Aplicado.

http://doi.org/10.17979/redma.2012.02.08.4761

Recibido: 21 Mayo 2012

Aceptado 8 Junio 2012

\section{RESUMEN}

En el mundo empresarial de la nueva era, la aplicación de las Tecnologías de la Información juega un papel fundamental en el desarrollo de estrategias de negocios eficientes. En este sentido, el Marketing de Afiliación o de Resultados, se ha convertido en una herramienta práctica, eficaz y útil al permitir medir y analizar las acciones de marketing llevadas a cabo por la empresa, lo que se traduce en mejoras, en un crecimiento sostenido y en un incremento del $\mathrm{ROI}^{3}$.

Bajo esta perspectiva, en este artículo lo que se pretende es analizar las ventajas e inconvenientes de esta herramienta online, haciendo especial hincapié en los modelos de pricing ó remuneración más empleados por las

\footnotetext{
${ }^{1}$ Doctora en Turismo por la UDC, Máster Oficial en Dirección y Planificación del Turismo por la UDC, Máster en Gestión del Medio Ambiente, Ecoturismo y Turismo Rural por la Universidad Complutense de Madrid, Técnico en Empresas y Actividades Turísticas por la Escuela Oficial de Turismo de Madrid, Profesora en el área de Comercialización en La Escuela Universitaria de Turismo de A Coruña, Profesoraen el Máster Oficial en Dirección y Planificación del Turismo de la Universidad de A Coruña.

${ }^{2}$ Doctora en Ciencias Económicas y Empresariales por la Universidad de A Coruña, Licenciada en Ciencias Económicas y Empresariales por la Universidad de Santiago de Compostela, Postgrado en Economía y Calidad en el Sector turístico por la Universidad de A Coruña, Profesora en el área de Comercialización e Investigación de Mercados en la Facultad de Economía y Empresa de la UDC, en La Escuela Universitaria de Turismo de A Coruña, y en el Máster Oficial en Dirección y Planificación del Turismo de la Universidad de A Coruña.

${ }^{3}$ ROI: Return On Investment
} 
empresas que la desarrollan. De la misma forma, intentamos aportar también algunas claves sobre los retos a los que se enfrentan en la actualidad y las tendencias futuras.

La metodología empleada se fundamenta, por las características propias del estudio, en la búsqueda de información y en su posterior análisis, a través de fuentes de información tanto primarias como secundarias.

Las conclusiones a las que hemos llegado a través de la investigación de esta herramienta permiten afirmar que el Marketing de Afiliación genera una oportunidad de ganar dinero sin necesidad de realizar grandes inversiones en logística, sin obligaciones financieras ó de gestión administrativa por parte de la red de afiliados, con el añadido de que el resultado puede ser medible, a diferencia de otras estrategias publicitarias, tratándose por lo tanto de una acción comercial rentable.

Palabras Clave: Marketing, Afiliación, Pricing.

\section{ABSTRACT}

INTELLIGENCE IN BUSINESS AND THE AFFILIATION MARKETING

In the business world of the new era, the application of information technology plays a key role in developing effective business strategies. In this sense, the affiliate marketing or results, has become a practical, efficient and useful to allow measurement and analysis of marketing activities undertaken by the company, resulting in improvements in sustained growth and increased $\mathrm{ROI}$.

From this perspective, this article the aim is to analyze the advantages and disadvantages of this online tool, with particular emphasis on pricing models or pay more employees for companies that develop it. Likewise, we contribute also some clues about the challenges they face today and future trends. 
The methodology is based, on the characteristics of the study, searching for information and further analysis through information sources both primary and secondary.

The conclusions we have reached through the research of this toolare such that the affiliate marketing creates an opportunity to earn money without requiring more investments in logistics, no financial obligation or administrative management by the network members, with the addition that the outcome can be measured, unlike other advertising strategies, being therefore a profitable business action.

Key words: Marketing, Affiliation, Pricing. 


\section{INTRODUCCIÓN}

Vivimos en una era de importantes cambios en todos los ámbitos en la que, sin duda, la gran revolución se está produciendo a nivel social y, sobre todo a nivel tecnológico. Hablamos de una era en la que se producen cambios exponenciales, donde la revolución tecnológica ha propiciado que el ayer, además de pasado, se haya convertido en algo obsoleto, caduco por lo que se hace necesario la aplicación de nuevas herramientas y nuevos modelos de negocio que ayuden a las empresas a ser, tal y como lo explican Rocco, E. y Dagnino, J (2009), Coopetitors ${ }^{4}$ (cooperate \& compite), es decir, más competitivos en el mercado. Estamos por tanto en la era de la afiliación.

Ante esta situación la estrategia lógica de las empresas debería basarse en estar presentes en todos los soportes tradicionales a través de acciones convencionales, pero como no, por supuesto que también en nuevos canales de comunicación. Máxime cuando estas acciones convencionales no están arrojando buenos resultados, con bajos niveles de $\mathrm{CTR}^{5}$, pocas conversiones y como consecuencia de todo ello un coste de adquisición excesivamente alto $\left(\mathrm{IAB}^{6}, \mathrm{PwC}^{7}, 2011\right)$.

El hecho de combinar los diferentes canales de comunicación que las marcas tienen con sus clientes, es una tendencia ante la cual las empresas de diferentes sectores se están posicionando, asumiendo que sus esfuerzos de comunicación deben diversificarse, y en esta dirección se encuentra el Marketing de Resultados.

\footnotetext{
${ }^{4}$ Coopetitors: Palabra anglosajona surgida de la unión de dos términos, cooperate \& compite. Hace referencia a la realización de una estrategia de cooperación competitiva entre diferentes autores (empresas, proveedores, clientes, socios...), que resultaría altamente ventajosa para todos.

${ }^{5}$ CTR: Click Through Rate

${ }^{6}$ Internet Advertising Bureau (IAB): Asociación líder en el sector publicitario interactivo.

7 PricewaterHouseCoopers (PwC): es la mayor firma de servicios profesionales del mundo. Ofrecen a las empresas y a las Administraciones servicios profesionales especializados en cada sector
} 
El Marketing de Afiliación o de Resultados, es una forma de promocionar, comercializar y distribuir productos y negocios por Internet mediante el cual un afiliado recibe una comisión por cada visitante, registro, cliente o venta que resulte de sus esfuerzos. La red de afiliados más simple consta de un solo vendedor y sus afiliados. Los afiliados se inscriben directamente a través de las plataformas, que son las responsables de la administración de los programas y del desarrollo técnico. En el año 2001 existían ya más de un millar de estos programas (Silverstein, 2001), de los que Amazon.com ya era el más grande en el mismo año (Bruner, Harden y Heyman 2001).

Después de que Tim Berners en el año 1989 reinventara y revolucionara la "triple www" a través de lenguajes como el $\mathrm{HTML}^{8}$ y nuevas tecnologías como el $\mathrm{XML}^{9}$, y de que en el año 1994 naciera amazon.com, las bases de la venta por Internet se habían sentado y con ellas, el modelo de afiliación.

Poco después nace la primera Plataforma de Afiliación, es en el año 1997, cuando Affilinet aparece en Alemania, aunque no sería hasta 1999 cuando, en Suiza, surge la que es en el año 2012 una de las primeras plataformas en España, Tradedoubler. Cabe precisar que en el año 2000 nace Zanox, de nacionalidad alemana, a la que le seguirían muchas otras iniciativas a nivel mundial.

\section{VENTAJAS E INCONVENIENTES DEL MARKETING DE AFILIACIÓN.}

Aunque a Amazon la experiencia de la afiliación le resultara exitosa y rentable, quizá deberíamos plantearnos la siguiente reflexión, ¿Por qué es importante el Marketing de Afiliación hoy como herramienta para obtener éxito comercial y, por extensión, empresarial?

\footnotetext{
${ }^{8}$ HTLM: Hyper Text Markup Language

${ }^{9}$ XML: Extensible Markup Language
} 
Los argumentos que podíamos esgrimir a favor son múltiples y variados, tanto para los anunciantes como para las redes de afiliados, aunque los más destacados podrían ser:

- Capacidad de ofrecer productos y servicios adicionales a los visitantes de las páginas, lo que genera una oportunidad de ganar el dinero en nuevos nichos de mercado gracias a ventas cruzadas.

- Oportunidad de obtener beneficio sin necesidad de hacer grandes inversiones en logística, sin obligaciones financieras, o de gestión administrativa por parte de la red de afiliados.

- No hay costo al afiliado hasta que se logren los resultados pactados, ya que el comerciante concede una comisión, en la mayor parte de los casos, sólo por las ventas que se hayan realizado.

- Es una acción comercial rentable, ya que algunas estimaciones hablan de que del sector global de Marketing de Afiliación se obtuvieron en el año 2006 entre $\$ 400$ - \$500 (miles) hasta alcanzar los $\$ 19.116$, aunque también es cierto que en el segundo trimestre de 2011, debido a la coyuntura económica mundial han perdido cuota de mercado y rentabilidad, (ValueClic ${ }^{10}$, 2011). Por otro lado, entre el 33\% y el 34\% de las ventas de un vendedor típico de productos por Internet viene a través de los afiliados (MarketingSherpa ${ }^{11}, 2011$ ).

- La publicidad está dirigida a los nichos más indicados. Ya que la información que el afiliado recibe está dirigida expresamente a él, en función de sus necesidades.

\footnotetext{
${ }^{10}$ ValueClic, Inc,(Nasdaq: VCLK) es una de las mayores compañías de marketing digital en el mundo. A través de una combinación única de datos, tecnología y servicios, ValueClic aumenta la conciencia de marca y la adquisición de unidades de atención al cliente en la escala de los anunciantes más grandes del mundo y maximiza los ingresos por publicidad de decenas de miles de editores en línea y móvil. Incluye en sus empresas Commission Junction (plataforma de Afiliación).

${ }^{11}$ MarketingSherpa: Se trata de una firma de investigación especializada en el seguimiento de lo que funciona en todos los aspectos de comercialización (y lo que no). Su objetivo es dar a los vendedores de todo el mundo las estadísticas, la inspiración y las instrucciones para mejorar sus resultados
} 
- Permite obtener resultados medibles sobre la publicidad de sus productos y servicios a diferencia de otras acciones comerciales, como por ejemplo, una cuña publicitaria en radio.

- Prolonga el alcance de la marca lo que provoca más visitantes de calidad, y más ventas a tantos afiliados como miembros tenga la red.

- Es un modelo de negocios por Internet que se traduce en diferenciación para la empresa.

- La aplicación de las nuevas tecnologías desarrolladas por los anunciantes y las redes de afiliación producen innovaciones en las páginas de afiliados.

- Para los afiliados, la afiliación puede suponer una forma de no depender exclusivamente de Google Adsence para hacer publicidad.

- En algunos casos los afiliados pueden rentabilizar las impresiones que no han podido vender a anunciantes directos.

Aunque los beneficios que hemos señalado parecen de peso más que suficientes para embarcarse en la aventura del Marketing de Afiliación, existen voces discrepantes, detractores que exponen sus argumentos en contra y que consideramos necesario destacar. Así podemos señalar como más significativos:

- Algunas empresas "poco éticas" utilizan artimañas para promover sus programas de correo basura o crean múltiples sitios Web con el propósito de generar tráfico artificial hacia los motores de búsqueda. Como ejemplo de esta situación podemos señalar lo ocurrido en Julio de 2008 cuando los grandes grupos informáticos, como Microsoft, Cisco y Sun Microsystems, tuvieron que distribuir, a través de sus actualizaciones automáticas de sistema, un patch ${ }^{12}$ para subsanar un importante fallo de seguridad en Internet. Este patch buscó recomponer un "agujero" que permitía a los piratas informáticos redirigir al usuario a una Web falsa, incluso aunque éste escribiera la dirección correcta, para obtener sus contraseñas bancarias (phishing).

${ }^{12}$ Patch: software de corrección 
- Los sitios Web que casi en exclusiva se desarrollan a través de enlaces de afiliados se ven con negatividad puesto que no ofrecen, habitualmente, contenidos de calidad.

- La mayoría de la gente no compra durante su primera visita a un Web site, aunque las cookies depositadas por los usuarios al navegar por Internet pueden asegurar que una referencia esté registrada. De este modo si la persona referida hace una compra en una visita futura, la empresa afiliada que la refirió originalmente mantiene el derecho de recibir una comisión por esa venta, es lo que se denomina contabilización de conversiones.

- Las duplicidades entre diferentes canales. Normalmente un mismo proveedor tiene campañas en paralelo en diferentes soportes y por tanto, tags de cada soporte repetidos en la pantalla de confirmación de compra. Esta es la razón por la que muchas empresas no están de acuerdo con este modelo.

- Encontrar buenos afiliados y que además sean rentables.

- Los fees de entrada y los mensuales fijos, hasta de $600 €$, que el anunciante tiene que pagar a algunas plataformas para poder entrar en los programas y beneficiarse de ellos.

- El pago a los afiliados se realiza, de manera casi habitual en todas las plataformas, entre 60 y 120 días. La demora en los pagos se produce porque en ese tiempo el anunciante puede comprobar si las cifras de ventas o registros son correctas.

- El fraude, proteger la marca del anunciante, sobre todo en los buscadores, son otros aspectos negativos destacados por los detractores. 


\section{PRINCIPALES MODELOS DE PRICING Ó REMUNERACIÓN EN EL MARKETING DE AFILIACIÓN.}

Tras lo expuesto con anterioridad, el Marketing de Afiliación puede ser considerado como una forma de franquicia digital o como un negocio de riesgo compartido, en el que a través de un sitio Web y de enlaces electrónicos a sus empresas afiliadas, el comerciante concede un pago por resultados, normalmente a cambio de ingresos de nuevos clientes a través de su Web site, por la suscripción a su servicio o por las compras que se generen vía estos enlaces.

Por este motivo, para esta herramienta la capacidad de medición que aporta el canal online donde se desarrolla, se transforma en algo básico y fundamental; ya que estas métricas le permiten al anunciante tener un control exhaustivo y en tiempo real tanto del coste de adquisición máximo, es decir, del coeficiente obtenido de dividir las comisiones totales entre el número de ventas realizado, como de su $\mathrm{ROI}$ ó lo que es lo mismo del resultado obtenido entre el valor de las ventas generadas y las comisiones totales otorgadas, y por tanto saber lo que les está retribuyendo a sus afiliados, y en definitiva lo que ganan.

En relación a las retribuciones que ofrecen los anunciantes, éstas deben ser competitivas y justas, sobre todo en las primeras etapas del lanzamiento del programa; ya que si la retribución es baja, es muy probable que la campaña no tenga éxito, genere poco volumen y por tanto los afiliados se sentirán mal retribuidos por su trabajo. Por eso los expertos aconsejan tener en cuenta la posición e intereses de los afiliados para marcar las comisiones, porque ellos evalúan sus ganancias por cada clic entregado (EPC. Earnings Per Clicks). Si no se tienen en cuenta estas premisas a la hora de retribuir a los afiliados la consecuencia será que no estarán satisfechos con el programa y a la larga resulte poco rentable y esto acabe con la campaña. 
Tal y como se publicaba en el Libro Blanco. Guía de Marketing de Afiliación de la IAB Spain (2010), en relación a las comisiones, "...Las comisiones deben ser utilizadas de forma táctica para fomentar la adopción del programa por parte de los afiliados, y después para manejar el crecimiento del programa (pensar a largo plazo). También existe la posibilidad de estructurar las comisiones para recompensar volumen y calidad por separado".

A continuación se exponen, a través de un cuadro resumen, los principales modelos de remuneración con los que trabajan la mayoría de Plataformas.

\section{Cuadro 1.- Principales Modelos de Pricing}

CPA: Coste por acción: se remunera el perfeccionamiento de una operación comercial. Ej: compraventa de bienes, arrendamiento de servicios, licencias de uso...

CPM: Coste por mil impresiones. se remunera la "impresión", con un tamaño determinado, de ese anuncio en un posicionamiento.

CPC/PPC: Coste por clic / Pago por clic. se remunera la activación de un enlace que eventualmente redirige a la página de destino que ha fijado el anunciante, independientemente de que el usuario acabe realizando alguna compra o acción en la Web o no la haga.

CPL: Coste por registro ó Lead: se remunera la cumplimentación de un formulario de datos, de contacto ó registro.

CPUV: Coste por visitante único: sistema cualificado de CPC en el que el "clic" es validado por múltiples filtros. Ej: clic-to-clic time, IP del país, referencia URL.

HYBRID: Sistema híbrido ó mixto: se remunera diferentes acciones, el híbrido más común CPA/CPL, aunque también se puede dar un CPC/CPM.

FIJO: Pago de un importe concreto por un posicionamiento ó integraciones determinadas durante un tiempo preestablecido .

Fuente: Elaboración propia

Como podemos observar existen diversas modalidades de asignación de resultados en el Marketing de Afiliación, siendo de vital importancia para 
ello los sistemas de tracking (rastreo) que permiten realizar el seguimiento de la navegación del usuario al que se ha marcado.

De todos estos modelos de remuneración, los que más beneficios están aportando a los afiliados y por lo tanto tienen más éxito son: el CPC/PPC, el CPL, el CPA, el CPM y el Pago Fijo Mensual.

Cuadro 2.- Principales Modelos de Remuneración

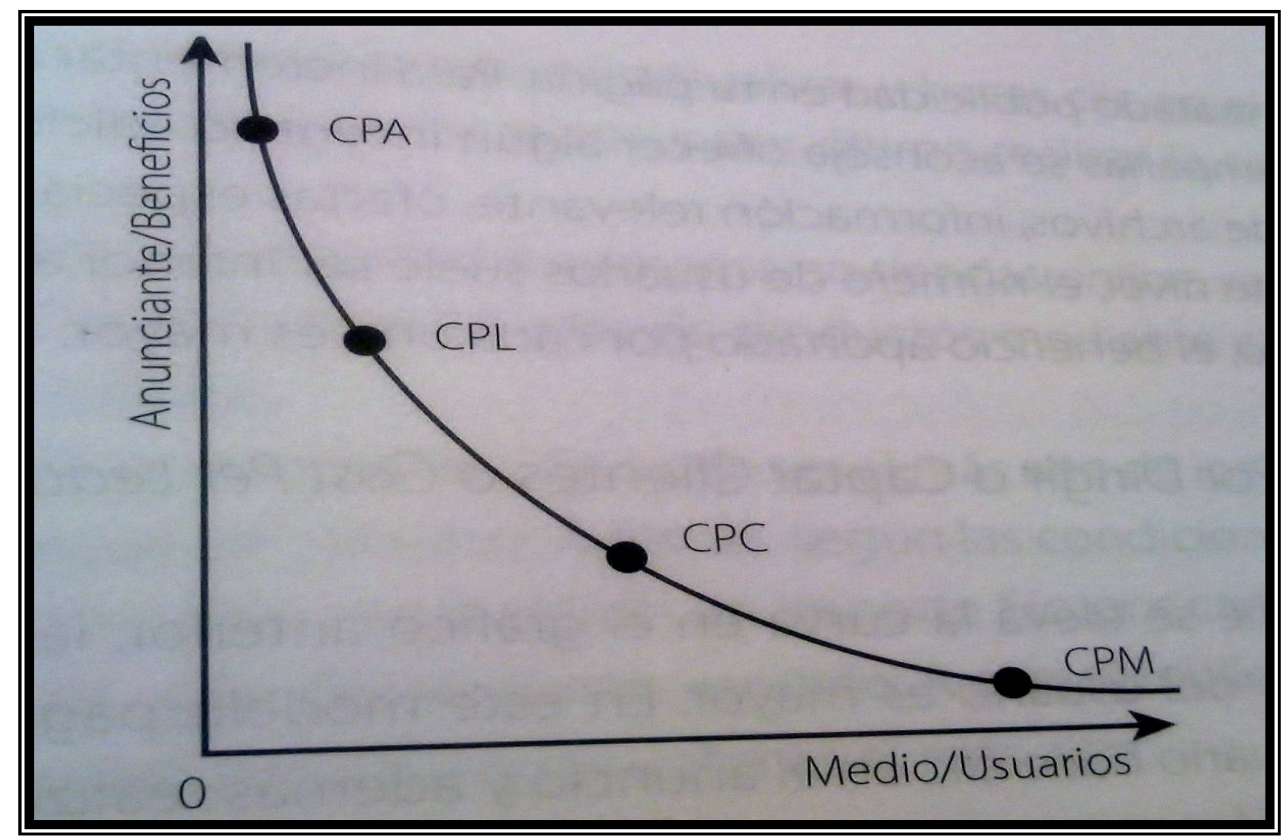

Fuente: Cerezo (2009)

Por la relevancia que estos sistemas de remuneración tienen en los programas, vamos a analizarlos más detalladamente:

\section{CPC/PPC: coste por clic/pago por clic}

Este sistema remunera la activación de un enlace que eventualmente redirige a la página de destino que ha fijado el anunciante, independientemente de que el usuario realice alguna compra o acción en la Web ó no. Se trata del modelo utilizado por Google Adsense en sites y Adwords en buscadores; así, por ejemplo, si un anunciante que vende vuelos online se publicita con las palabras clave "compañías aéreas" la esperanza 
de que un cliente potencial que esté buscando vuelos vea el anuncio, haga clic en él y sea redireccionado a la Web del anunciante son muy altas; y aunque puede ser que en esa visita no compre, el anunciante habrá conseguido una oportunidad de venta que puede reportar un importante beneficio en un futuro más ó menos próximo.

El coste por clic es una de las mejores formas de orientar productos a un mercado específico a un costo relativamente bajo, al mismo tiempo que es una buena opción cuando el objetivo es atraer visitas a una Web. Los anunciantes tan sólo realizan el pago de un canon sobre la base de su oferta cuando se hace clic en su anuncio y aunque parece poco adecuado para las pequeñas empresas, el éxito parece asegurado independientemente de sus dimensiones.

Expertos de la afiliación como Cerezo (2009) indican que “...para incrementar el éxito de este tipo de campañas se aconseja ofrecer algún incentivo adicional al usuario: descarga de archivos, información relevante $u$ ofertas especiales."

En este modelo el número de usuarios suele ser menor que los del modelo CPM, tal y como se puede apreciar en el cuadro superior, sin embargo, el beneficio aportado por cada uno es mayor.

Dentro de este sistema de remuneración existe lo que se denomina "Redes de Anuncios CPC". Estas Redes están configuradas por las más importantes redes de publicidad en línea; en la actualidad a la cabeza de cualquier ranking encontramos a AdWords de Google y Yahoo! Search Marketing (anteriormente conocido como Overture). A través de estas redes existen dos tipos de entidades que pueden ser las beneficiadas: los anunciantes y los afiliados.

Los anunciantes tienen varias opciones para configurar una campaña de publicidad y llegar a su mercado objetivo en la Web. Una de ellas es a través de "palabra clave", aunque tal y como escribe Stokes (2010), 
fundador de AdGooroo empresa líder en motores de búsqueda, con más de quince años de experiencia en publicidad y gestión en Internet, tan sólo el $3 \%$ de la publicidad a través de este sistema obtiene importantes beneficios para estos anunciantes; otra opción sería un anuncio de un producto o de un servicio de redes CPC.

Un anuncio de un producto de redes CPC permite a sus anunciantes enviar de manera gratuita un feed (tipo de dato empleado para suministrar información que es actualizada con frecuencia) de sus productos a la base de datos de la red de anuncios. El problema surge cuando una persona busca un producto específico y se encuentra con diversos enlaces para diferentes anunciantes de productos similares.

Cabe precisar que los anunciantes que pagan para que aparezca un producto por lo general aparecen en la parte superior de los resultados. Sin embargo, esto no garantiza el éxito ya que una vez que los productos de muchos fabricantes están listados en la pantalla, el espectador puede fácilmente decantarse por un producto por criterios como, por ejemplo, el precio. Es frecuente encontrar estos sitios con el nombre de "motores de comparación de productos" ó Metabuscadores.

Los anuncios de servicio de redes CPC son muy similares a los productos CPC, excepto que permiten a sus anunciantes enviar un feed de su servicio a la base de datos de la red. Los servicios más populares pueden incluir pasajes de avión, habitaciones de hotel, etc.

Al igual que los anunciantes obtienen ventajas de esta forma de publicitar y comercializar sus productos, los editores que desarrollen sitios Web también pueden beneficiarse. Obtener rentabilidad de casi cualquier tipo de contenido es bastante fácil con esta fórmula.

Del mismo modo que hay muchas redes de anuncios para que los anunciantes puedan promocionar sus productos, existe también una amplia gama de redes que hacen que sea extremadamente fácil para los afiliados 
rentabilizar sus sitios Web, como ocurre con AdSense de Google, Yahoo! Ó Editores Red. Cada vez que un cliente hace clic en un anuncio de los editores del sitio, la red publicitaria paga una comisión. Muchas redes ad no dan detalles exactos sobre la cantidad que van a pagar por cada clic en anuncios, sin embargo las estimaciones varían entre el $10 \%$ y el $70 \%$.

A continuación se propone un ejemplo de cómo funciona esta forma de remuneración.

\section{Cuadro 3.- Ejemplo remuneración CPA}

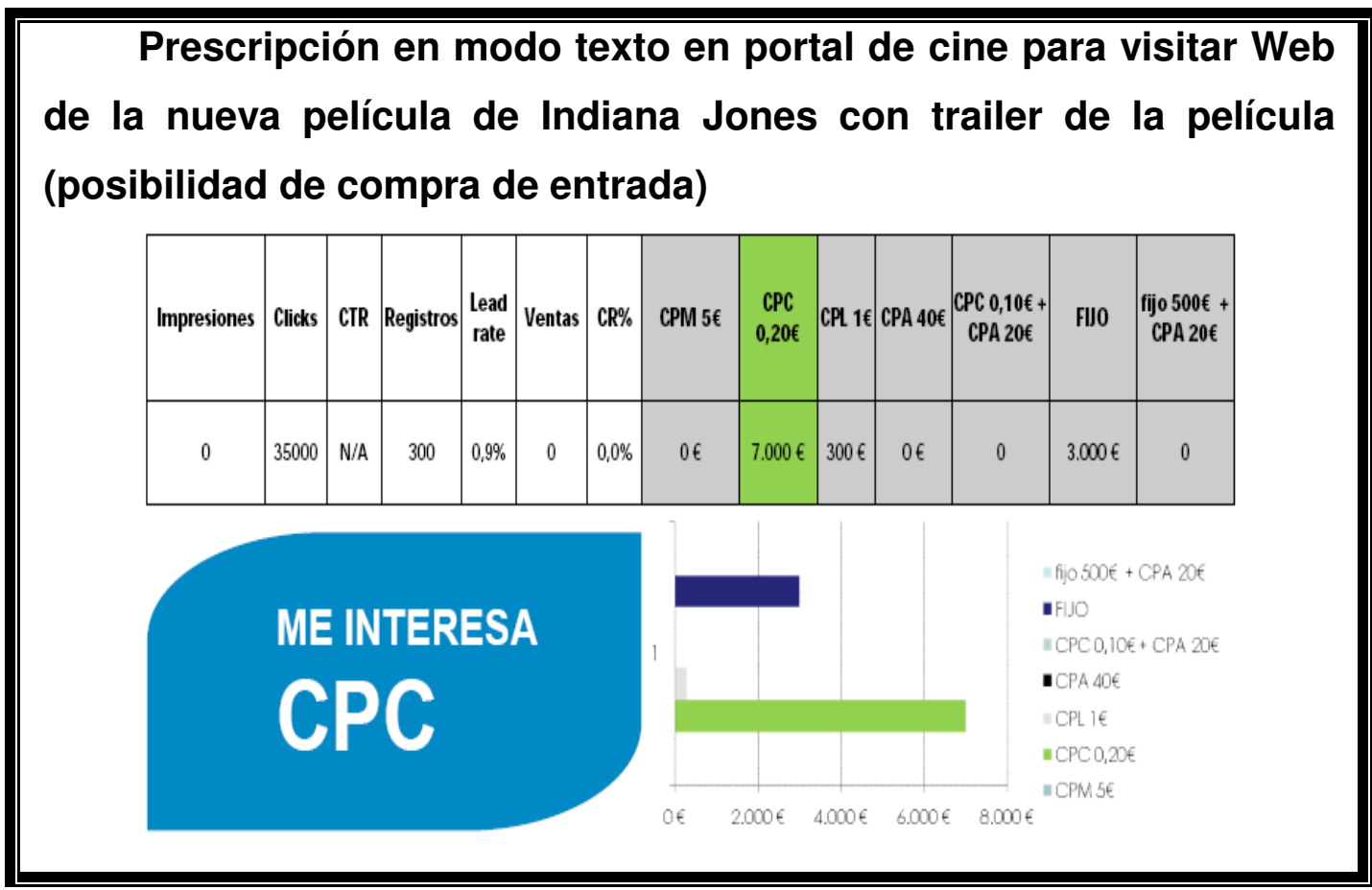

Fuente: adaptado de Murillo. J (2008)

Según la encuesta realizada por RentabilizarWeb ${ }^{13}$, las 4 mejores Plataformas de Afiliación 2011 de CPC, fueron las siguientes: hi Hi-Media, $\mathbf{B}$ Bin-Layer, $\oplus$ Mooxar y por último \& Smowtion-Media.

\footnotetext{
${ }^{13}$ RentabilizarWeb: la primera comunidad oficial de afiliación española.
} 


\section{Cuadro 4.- Mejores Plataformas de CPC 2011}

\begin{tabular}{|c|c|c|c|}
\hline Interfaz: & Interfaz: & Interfaz: & Interfaz: \\
\hline Comunicación : 6.6 & Comunicación : 6.8 & Comunicación: $\quad 7,3$ & Comunicación: 7.8 \\
\hline Rendimiento: 6.5 & Rendimiento : & Rendimiento: & Rendimiento: \\
\hline Pagos: & Pagos : & Pagos & Pagos: \\
\hline Evolución: & Evolución : & Evolución & Evolución: \\
\hline Nota final: 6.7 & Nota final: 6.4 & Nota final: 7.3 & Nota final: 6 \\
\hline
\end{tabular}

Fuente: adaptado de Rentabilizar-Web (2011)

\section{CPA: coste por acción/coste por adquisición}

En este sistema el anunciante paga por acción en la Web después de haber hecho un clic en un anuncio. Esta acción puede ser un registro, una compra ó cualquier acción medible que el anunciante elija. Para aquellos interesados en obtener beneficios de su sitio Web o que tienen un producto 0 servicio que le gustaría anunciar y promover, CPA se considera una de las más ventajosa formas de publicidad disponible en el canal online.

Cabe señalar que el anunciante por lo general, determina qué tipo de acción va a pagar y pueden incluir el pago de un solo tipo de acción ó de varios tipos. Esta forma de remunerar es muy utilizada por sectores como el de los viajes, banca, seguros, telefonía, tecnología, moda ó gambling.

Si nos situamos en el gráfico de remuneraciones podemos observar que esta forma de pago se sitúa en la zona más alta, porque la acción que se le requiere al usuario es la mayor de todos los sistemas. En relación al precio de estas campañas podemos decir que es el más alto de los cuatro que vamos a desarrollar, ya que la complejidad del proceso es muy elevada. 
Al igual que existen las "Redes de Publicidad CPC", también están las "Redes de Publicidad CPA". El registrarse en una red CPA permite realizar promoción y comercialización de anunciantes, productos y servicios en un sitio Web. Los editores que firman por la red publicitaria CPA son los afiliados, por lo que las CPA ad Redes se denominan a veces redes de afiliados. Si bien esto no es del todo incorrecto, existen algunas diferencias entre ambos.

CPA ad Redes pueden considerarse como intermediarios CPA, que trabajan muy estrechamente con la publicidad de sus clientes para encontrar el beneficio de los editores y anunciar sus productos. Se diferencian de las redes de afiliados en primer lugar, en que las CPA ad Redes están muy centradas en los clientes mientras que la mayoría de las redes de afiliados son mucho más pasivas cuando se trata de CPA anunciante. Por otra parte, muchas redes de afiliados suelen exigir a sus miembros pagar una tasa de puesta en marcha, así como ser precalificados en una serie de ámbitos. Como editor, las CPA ad Redes suelen ser mucho menos estrictas a la hora de poder adherirse y normalmente son gratis.

Por otra parte, las CPA ad Redes en general trabajan arduamente en mantener a sus afiliados mediante el pago de buenas comisiones y un calendario de pagos regulares. La mayoría de las Redes ad CPA prestan especial atención a las campañas de los anunciantes a sus afiliados y éstos pueden obtener un trato preferencial sobre todo si producen una gran cantidad de ingresos o se encuentran en un nicho específico. Asimismo, cabe señalar que muchas Redes ad CPA suelen sondear otras campañas y otras redes de anuncios para ver si otros afiliados están interesados en promover una campaña específica. En la industria esto se conoce como competencia.

Hay que destacar que el CPA también puede ser Coste por Adquisición, si bien la generación de clientes es la principal función de la CPA, los afiliados también pueden promover y anunciar un producto en su página Web con el objetivo de ser vendido. Si este producto se vende, se supone que el 
usuario ya ha hecho un largo recorrido, ya que ha visualizado el anuncio, ha hecho clic en él, ha rellenado en algunos casos un formulario y finalmente ha comprado.

Si bien esta es una de las maneras más ventajosas para el mercado de un producto para un anunciante, hay algunos riesgos por parte del afiliado, tales como el gasto de promoción del producto y el espacio publicitario ocupado en su Web site. Por todo ello las Redes CPA trabajan muy estrechamente con el anunciante asegurándose de que el producto se adapta e interesa a sus afiliados. Existen multitud de pequeñas empresas que venden productos y servicios, una cosa que los afiliados deben considerar al pensar en unirse a una Red CPA son todos los grandes nombres de los anunciantes que utilizan este tipo de red. Se trata de un sistema que atrae hasta en un $40 \%$ a grandes empresas, por lo que quizás no sea lo más adecuado para las Pymes.

En el siguiente ejemplo podemos ver la realidad de este modelo de remuneración:

\section{Cuadro 5.- Ejemplo remuneración CPA}

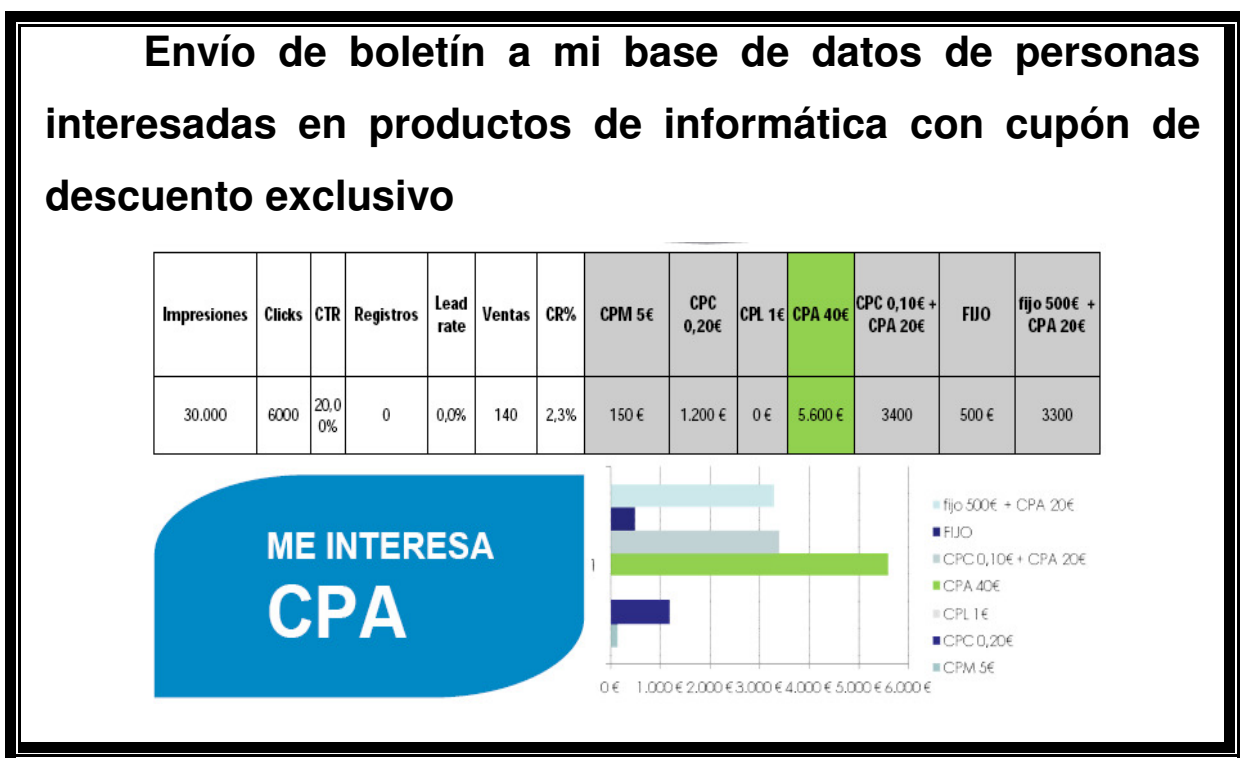

Fuente: adaptado de Murillo. J (2008) 


\section{CPL: coste por lead/coste por registro}

El objetivo de esta forma de remunerar es conseguir contactos cualificados, los cuales se pueden convertir en clientes y, en un futuro, en ventas. Este sistema establece el pago de una cuota cuando un usuario solicita más información a través de un formulario ó cuando hace una suscripción al boletín electrónico del anunciante, por lo que la acción que se le requiere al usuario es mayor, lo que provoca que la posición en la curva del gráfico anterior, de esta manera de remunerar, sea más elevada.

Es recomendable que esos formularios soliciten datos de contacto como el correo electrónico ó el teléfono, ya que a posteriori se podrán utilizar estos datos para enviar información u ofertas que se ajusten a las necesidades de cada usuario, cumpliendo el requisito de contar previamente con su permiso, y por lo tanto no vulnerar la Ley de Protección de Datos.

La cuantía de este modelo suele ser superior a la del resto, ya que el tiempo que se requiere desde que el usuario accede a una página hasta que termina en la del anunciante y cumplimenta el formulario es mayor.

A este nivel el número de usuarios disminuye, sin embargo en contrapartida, el beneficio aportado por cada uno de ellos es muy superior. Este sistema está recomendado para los sites de entretenimiento, ONGs salud y belleza, coches, banca y seguros.

El siguiente ejemplo nos ofrece una idea más clara de la utilidad de este sistema:

\section{Cuadro 6.- Ejemplo remuneración CPL}


Portal de videojuegos. Envío de boletín a mi base de datos (Registro de jugador existente WoW, regalo armadura exclusiva. Posibilidad de comprar ampliación Wrath of the Lich King)

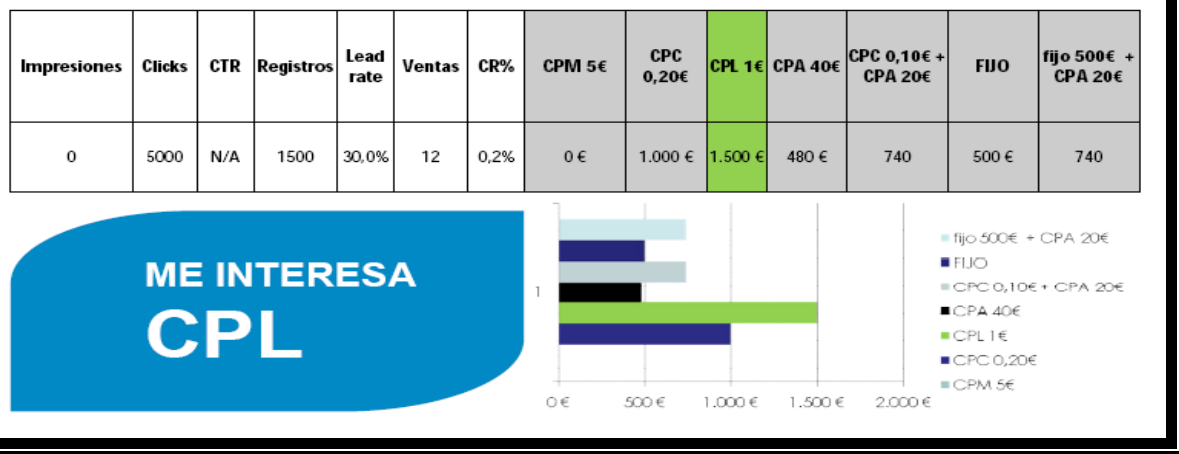

Fuente: adaptado de Murillo. J (2008)

\section{CPM: Coste por mil impresiones}

En este caso la remuneración viene ocasionada por el número de impresiones de un anuncio, es decir, el número de veces que se visualiza la publicidad en la página de un afiliado. Este sistema se recomienda cuando el objetivo que se persigue es conseguir un reconocimiento de una marca, es decir, conseguir visibilidad. En estas campañas, el número de usuarios suele ser muy elevado, por lo que el valor aportado por cada uno es menor. Está recomendado para cualquier tipo de anunciantes.

A continuación vamos a ejemplificar este modelo:

\section{Cuadro 7.- Ejemplo remuneración CPM}




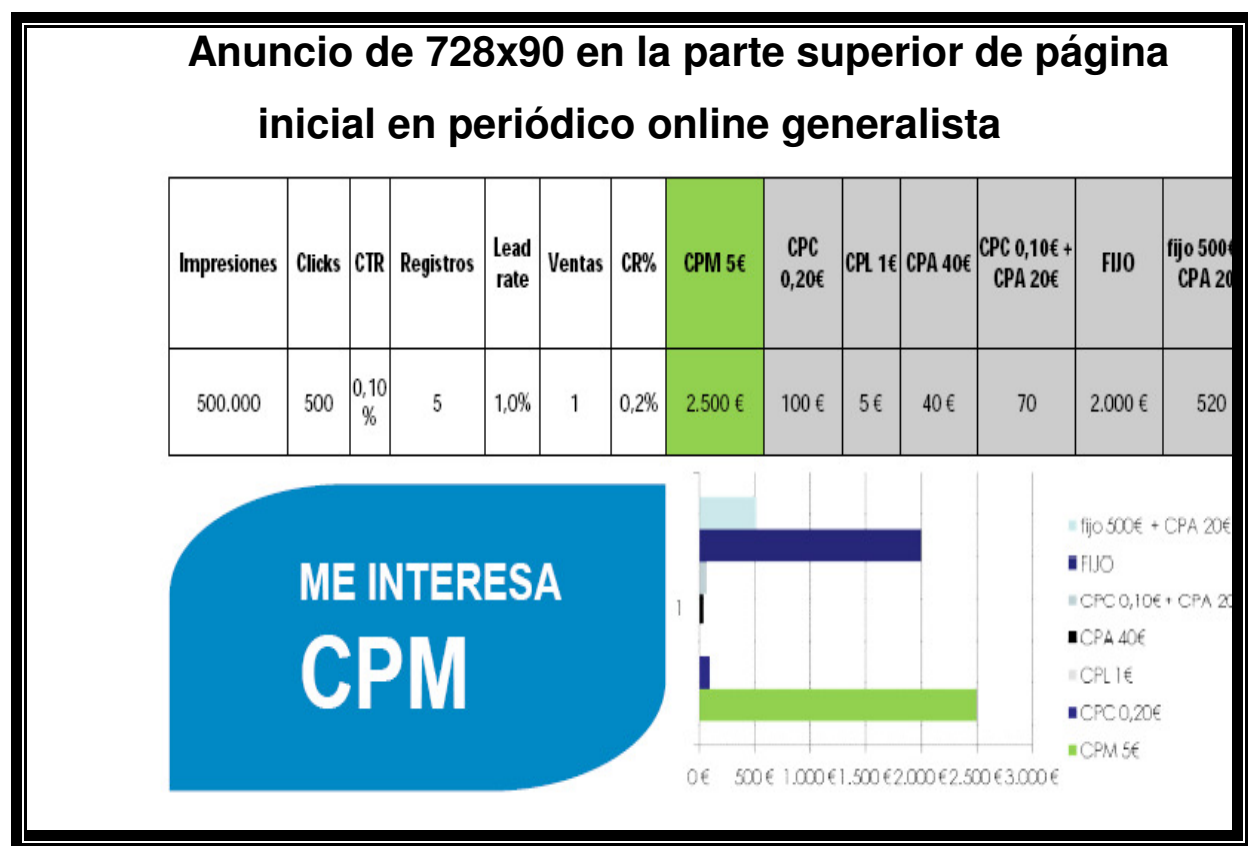

Fuente: adaptado de Murillo. J (2008)

\section{Pago Fijo Mensual}

Con este sistema los anunciantes pagan una cantidad fija al mes por su anuncio en las páginas solicitas. El precio de la remuneración se establecerá en función de las estadísticas y el perfil del site, su temática, el grado de especialización, visitas únicas diarias..."...se trata de un complemento al resto de modelos, porque permite planificar con más fiabilidad al ofrecer la posibilidad de comparar entre distintos modelos y así analizar cuál de ellos resulta más eficaz", Cerezo (2009).

Una vez analizados los modelos de remuneración más habituales parece claro que la elección del modelo más adecuado en cada caso estará condicionado por diferentes factores tales como: el tipo de empresa y de producto que se pretenda vender; las necesidades de la publicidad; el público objetivo y el potencial; el presupuesto del que se disponga y por supuesto los objetivos que se persigan.

Así pues, si un anunciante tiene como objetivo el branding y además incrementar sus ventas, el modelo más adecuado será un CPL ó CPA, por que con ellos el logro de estos objetivos será más fácil; aunque es cierto que 
con estos modelos los afiliados corren un riesgo mayor, ya que sólo van a obtener beneficios cuando algún usuario se registre o compre. Por este motivo, los afiliados tienden a posicionarse en los modelos de CPM y CPC, ya que con ellos sus posibilidades de obtener beneficios son mayores y los riesgos menores.

Parece necesario, llegados a este punto, incidir que en ocasiones los intereses de anunciantes y soportes son inversamente proporcionales, tal y como se puede apreciar en el cuadro siguiente:

\section{Cuadro 8.- Cómo monetizar un site}

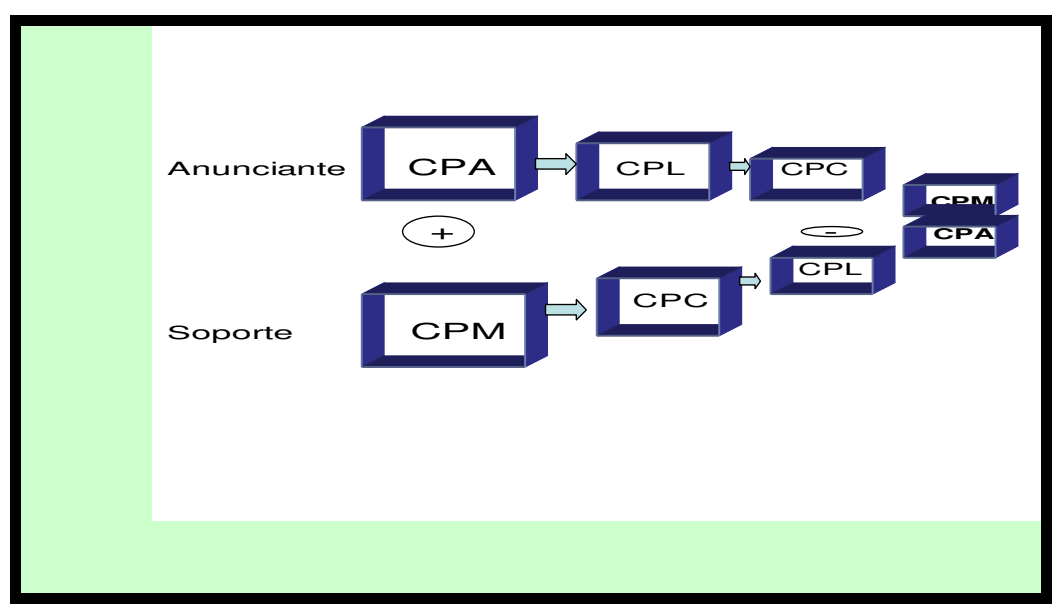

Fuente: Adaptado de Martínez.J (2009)

Como podemos observar, el anunciante rentabilizará en mayor medida su programa cuando su obligación de remunerar al soporte se propicie a través de cualquier acción que el cliente efectúa en su site (CPA) y en menor medida cuando se obtenga de las impresiones (CPM). Por el contrario, al soporte lo que le interesa en primera instancia es ser remunerado por el anunciante por impresiones (CPM), ya que en todo caso va a cobrar y en último caso por alguna acción que el cliente efectúe en el site del anunciante (CPA).

De esta forma, los expertos en afiliación recomiendan llevar a cabo campañas mixtas, híbridas, de diferentes modelos lo que permitirá analizar qué modelos funcionan mejor con determinados formatos de publicidad, 
mensajes publicitarios, ubicación en el site, herramientas. De igual forma el anunciante podrá conocer cuál es su público objetivo y conseguir un tráfico de calidad, lo que permitirá una relación "win to win".

Independientemente del modelo que se elija, durante toda la campaña $y$, gracias a las herramientas desarrolladas por las plataformas, el anunciante podrá medir el éxito de la misma a través del Clic Through Rate (CTR), que mide el número de clicks que se hace en un anuncio en relación con el número de impresiones del mismo. Cuanto mayor sea el porcentaje resultante de este coeficiente, más éxito habrá alcanzado esa campaña. Al final de la campaña este dato deberá ser comparado con los beneficios obtenidos para estimar la eficacia real de la misma, ya que, aunque el número de clicks sea elevado, eso no significa que todos los usuarios hayan efectuado una acción.

En el caso del afiliado y, para conocer la rentabilidad de una campaña, lo que tiene que analizar es el Coste por Mil Impresiones Efectivo (eCPM). Este ratio consiste en dividir nuestros ingresos procedentes de la red de afiliados y dividirlos entre las impresiones que hayamos utilizado entre mil. De este modo estandarizamos diferentes modos de pago de publicidad y cobros a una única medida (Jiménez, 2009). Lo que este autor explica es que "...si vendemos publicidad en nuestro site a CPM y tenemos un anunciante que nos paga a CPA, por medio del eCPM pasamos los ingresos de CPA a CPM, y de esta manera podemos comparar ingresos y gastos, con la misma unidad de medición".

Además de los inconvenientes propios de cada programa, en ocasiones surgen dudas sobre quién debe ser recompensado por el resultado obtenido, ya que muchas veces son varios los medios que prescriben un mismo producto/servicio. En estos casos las cookies permiten conocer mejor el "viaje" de una persona desde que conoce los productos, compara otros similares, $y$, finalmente, los adquiere. 
Otro aspecto interesante del que debemos de ser conscientes es el objetivo prioritario que como anunciante nos interesa conseguir con un determinado programa, ya que ¿es más valioso lograr dar a conocer un producto/servicio al público ó lograr que éste se interese, visite al anunciante y eventualmente, lo adquiera? Históricamente en las relaciones performancebased el más valioso ha sido aquel cuya cookie tenía la fecha mas reciente antes de la venta/acción.

Llegados a este punto quizá sería recomendable hacernos la siguiente pregunta: ¿qué factores hay que tener en cuenta a la hora de negociar estos modelos de remuneración?

Siguiendo las pautas de Javier Murillo (Publisher Group Sales Director de TradeDoubler) algunos elementos que deberían ser tenidos en consideración serían:

- Según la tipología del soporte del prescriptor: sitio de noticias, como por ejemplo un periódico online, portal vertical, sitio de compras, comunidad de compradores, blogs, comparadores de precio.

- La identidad/relación entre el contenido y el producto/servicio prescrito: idealmente por decisión humana; alternativamente por una solución integrada de anuncios contextuales td Admatch.

- La adecuación del perfil medio del visitante al producto/servicio prescrito: ¿productos financieros para menores de edad? ¿secciones temáticas específicas con prescripciones relacionadas?

- La calidad del copy/hook (modelo de autorregulación) y nivel de agresividad en la oferta (oferta válida sólo hoy, oferta sólo válida desde este enlace...).

- La confianza de los visitantes en las recomendaciones del propio soporte: ¿el público confía en lo que recomiendo? seguramente si lo dicen los responsables de un portal con credibilidad será bueno, voy a probarlo; si los recomienda Matías Prats es que en ING será bueno... 
- El nivel de integración/diversidad en la cartera de productos/servicios ofertados: no es lo mismo una tienda integrada con ordenadores de una marca que con ordenadores de diez marcas y tiendas diferentes.

- Exclusividad de las ofertas: ventajas exclusivas por registrarse desde esos enlaces específicos.

A continuación se exponen algunos ejemplos reales de cómo las empresas desarrollan sus modelos de remuneración en función de sus programas de afiliación. Se trata de cuatro anunciantes de ámbitos diferentes, las compras y subastas "eBay"; las relaciones personales "be2" y los dominios en Internet "Sedo".

\section{Modelo CPA y CPL: "eBay"}

"eBay" es una de las páginas más famosas de Internet. Disponible en multitud de idiomas y con presencia en varios países, permite la subasta de casi todo tipo de artículos que los propios usuarios se encargan de poner a la venta. Es famosa, sobre todo, por poner casi cualquier cosa a la venta y, en algunas ocasiones, a precios más que asequibles. Su propio eslogan es un fiel reflejo de esta política: “¿No encuentra lo que busca? Búsquelo en eBay”.

Además eBay permite el pago de los objetos adquiridos mediante PayPal, que pertenece a la misma compañía y es uno de los métodos más seguros y extendidos de pago por Internet que existen. Miles de usuarios ofrecen multitud de artículos cada día y su tasa de crecimiento es de las más altas de Internet, lo que la convierte en líder del mercado electrónico en muchos países.

\section{El programa de afiliación}

Ser afiliado de eBay es una opción gratuita y segura, ya que se trata de una empresa internacional y muy conocida. El afiliado cobra hasta $14 €$ por cada nuevo usuario activo que se registre (entre $8.5 €$ y $14 €$ como máximo) y desde $0.10 €$ hasta $0.19 €$ por cada puja que se realice en las Webs durante el mes en activo y dependiendo del volumen generado. 
Este anunciante ofrece herramientas especiales para sus afiliados y un equipo para la resolución de problemas. Son socios de Commission Junction y TradeDoubler. Disponen de banners, enlaces de texto, integraciones de contenido, correo electrónico para la base de datos de sus usuarios, etc.

Para controlar las ganancias, los afiliados disponen de un panel de control donde pueden medir y consultar los diferentes informes que se les ofrecen.

- Ventajas del programa: mucha flexibilidad a la hora de diseñar la estrategia, incluso disponen de una API para desarrollar la Web orientada a la venta de artículos por eBay. Tienen mucha información para el programa de afiliación.

- Inconvenientes del programa: Hay mucha competencia, sobre todo en el modelo de afiliación mediante la compra de palabras para adwords y similares.

\section{Modelo CPC, CPA y CPL: "be2"}

"be2" es una empresa de origen Alemán, presente en más de 25 países. Se dedica al mercado por Internet de las relaciones personales estables. La diferencia que ofrecen en be2 respecto a otras empresas del mismo tipo, es que garantizan encontrar a personas afines a tu personalidad de acuerdo a un método científico desarrollado por ellos mismos y que analiza la compatibilidad entre dos personas mediante criterios psicológicos, antropológicos y sociológicos.

Cuando un usuario se registra en be2 recibe un completo perfil y una descripción de la que podría ser su pareja ideal en función de su compatibilidad. Además cada candidato recomendado recibe el índice de compatibilidad atendiendo a los datos, características, opiniones e intereses. Una completa evaluación de los apartados y un gráfico tanto de similitudes como de diferencias.

\section{El programa de afiliación}


El programa para afiliados de be2 está gestionado directamente por Tradedoubler. Las herramientas de promoción de las que disponen son gráficas, con banners.

Es importante considerar que sólo se contarán como registros, clicks y ventas válidas aquellas que procedan de España, por lo que la IP del usuario ha de ser española. No se incluyen IPs de otros países de habla hispana o de otros países europeos.

Las comisiones cobradas son diferentes en función del objetivo conseguido. Así, si se consigue un clic éste reportará al afiliado $0.05 €$, con un registro se consigue $1.5 €$ y por último, la parte más difícil, la venta de una suscripción que pueden ser de 3, 6 ó 12 meses, que aportará a la cuenta del afiliado $25 €$.

- Ventajas del programa: Buenas comisiones con los registros. Admite comisiones por clicks.

- Inconvenientes del programa: Este tipo de programas no goza de mucha popularidad entre clientes y afiliados.

Aunque este tipo de programas no gozan de buena reputación entre afiliados, que se quejan de la poca fiabilidad de los datos, y entre los usuarios, que se quejan de la dificultad de darse de baja o cancelar sus datos de una cuenta, sigue siendo uno de los contenidos más visitados en Internet. Cada año millones de personas se apuntan a este tipo de páginas Web, entre las que destacan unas 10 compañías en el mundo y be2 es una de ellas.

\section{Modelo CPC, CPA: "Sedo"}

Cada día se registran en todo el mundo miles de dominios y "Sedo" es un referente en el mundo de los dominios. Ofrece diversas herramientas a los domainers, destacando el sistema de parking de dominios. Pero no sólo dispone de un servicio de parking de dominios sino que ofrece diferentes 
servicios de alto valor añadido como un servicio de valoración, transferencia, venta y subasta de dominios.

El servicio de parking de Sedo está disponible en 20 idiomas, por lo que la gran parte de los usuarios de Internet pueden ver sus enlaces en su propia lengua, dando lugar a un incremento de clicks y un mayor número de ingresos para los usuarios de Sedo.

\section{El programa de afiliación}

El programa de afiliación ofrece herramientas que el afiliado puede incluir en su página Web y que tras su utilización le redirigirá a Sedo, donde si la visita se transforma en compra el afiliado podrá ganar diferentes comisiones por venta.

El programa de parking no está incluido directamente en el programa de afiliados, pero puede ser otra forma de conseguir ingresos por éstos, aunque en este caso los dominios no estarán desarrollados sino que los dominios apuntan a los servidores de Sedo, bien mediante DNS, bien mediante redirección; y nos aparecerá una pantalla con enlaces relacionados con la temática de nuestro dominio. Por cada click el afiliado recibirá una pequeña comisión. Al igual que en el programa de AdSense de Google, esta cantidad dependerá de lo que el anunciante haya invertido y de la temática.

Respecto a las secciones que sí ofrecen en el programa de afiliación, poseen un buscador de dominios, que muestra los dominios ya registrados a la venta en Sedo, un servicio de asesoramiento en negociaciones, un servicio de transferencia de dominios, recuadros con los escaparates de dominios o de las últimas ventas realizadas.

La comisión siempre será un fijo, el $25 \%$ de la comisión que Sedo se lleve por la venta o la operación, y en el caso de asesoramiento añaden un plus de $17,25 €$ por solicitud. 
En general las herramientas se basan en tamaños de banners ya conocidos o en algunos formatos especiales para las cajas de búsqueda o de escaparate. Al utilizarse un sistema de link de afiliado, es decir, un link con un código de afiliado único, se pueden utilizar enlaces directos desde el contenido de la página Web del afiliado, que pueden ser mucho más beneficiosos en caso de escribir textos con temática de dominios o Webs.

Si el afiliado tiene una Web relacionada con alojamiento, diseño, Webs, etc. es un complemento ideal para el programa de publicidad.

- Ventajas del programa: Es un servicio muy conocido y respetado por el mundo de los dominios.

- Inconvenientes del programa: La conversión de las ventas ha de realizarse en la visita sobre el banner, no hay un margen de tiempo.

\section{RETOS Y TENDENCIAS DEL MARKETING DE AFILIACIÓN}

Como hemos visto, en la cadena de valor de la publicidad digital existen diferentes agentes que aportan soluciones y métodos para optimizar la relación entre anunciantes y el medio online. Estamos, sin duda, en un momento de búsqueda de la eficiencia, intentando mejorar la imagen de las marcas y maximizando los resultados obtenidos a través del canal online, tanto desde el punto de vista del anunciante, como desde el del Publisher ó afiliado, al buscar la mejor rentabilidad de los espacios publicitarios.

Pero para conseguir estos objetivos, hay que salvar retos. Retos a los que se enfrenta el Marketing de Afiliación, condicionados a su vez por las tendencias y por el desarrollo, tanto tecnológico como social. Estos retos y tendencias los podríamos resumir en los siguientes puntos: especialización de las plataformas; llevar a cabo estrategias de partner; conseguir afiliados premium; nuevas políticas de pagos; incrementar el no de players y realizar una apertura al exterior; crear formatos más creativos; aumentar la colaboración y transparencia (Valdivielso, 2010).

A continuación se explican estos aspectos de forma más detallada: 
Especialización de las plataformas. En plena era digital, el mercado está cada vez más segmentado, lo que permite atender a cada cliente de una manera más especializada, más personalizada y satisfacer sus necesidades de forma más efectiva. Pero esta realidad, de momento, es aplicable a muy pocas plataformas de afiliación. Así, Zanox, es una de las pocas que tiene una división de viajes, entre otras, y por lo tanto está especializada en este mercado, lo que le confiere una clara ventaja frente a sus competidores. La tendencia en este sentido es especializarse tanto en modelos de negocio como en sectores, para conseguir una mayor eficiencia en los programas.

Estrategias de partner. Para aquellas empresas nuevas en el medio, es fundamental que las plataformas sepan asesorar y guiar en el mundo de la afiliación a sus clientes, tanto anunciantes como afiliados, para que éstos sepan elegir. La tendencia, por pura lógica y necesidad, es caminar hacia una empresa con vocación de partner, de colaboración en el largo plazo y que esté en toda la cadena de valor. Conocer a los clientes, sus productos, estrategias, rentabilidades, objetivos de marketing y comunicación, son cosas que no se aprenden en el corto plazo, por lo que tiene que establecerse una relación de confianza, basada en el trabajo diario y la transparencia, desde el primer día.

La elección del partner correcto, es decir, de la red de afiliación, es esencial para el éxito de las actividades. La red es la plataforma para el programa y cuenta con el pool de afiliados de donde el publisher puede seleccionar a los que mejor encajen con su estrategia. Es más, debe ofrecerle un seguimiento completo y el proceso de comisión para los afiliados, junto con todas las herramientas para la gestión del programa (informes, gestión creativa, aprobación y comunicación con afiliados, etc.). De cara a elegir la mejor red, el publisher debe tener en cuenta los siguientes puntos: experiencia en Marketing de Afiliación y Performance Marketing; el tamaño y la calidad del pool de afiliados; diversidad de modelos de negocio; número de programas en marcha y transparencia. 
Afiliados Premium. La situación coyuntural, económica y social, que se está viviendo desde el año 2008, ha hecho que el desarrollo de estrategias en el canal online cada vez cobre más relevancia. El Marketing de Afiliación ofrece a los anunciantes no sólo una publicidad online segmentada, sino sobre todo, la posibilidad de ventas online directas. A través de las páginas Web de sus afiliados, los anunciantes pueden beneficiarse rápidamente de una red de ventas virtual, haciendo que Internet se convierta en un canal de distribución muy efectivo.

Pero no sólo a través de páginas la afiliación se están desarrollando estos programas, cada vez más, las redes sociales se están convirtiendo en un modelo de negocio, lo que permite ampliar la tipología de clientes. La tendencia es a trabajar con afiliados premium, más sofisticados y que ofrezcan a los anunciantes productos acordes a sus sites de manera que las posibilidades de conversión en clicks o leads sean mayores, generen un tráfico más cualificado y moneticen más aún. Esto no sólo beneficia al afiliado sino también a los anunciantes.

Políticas de Pagos. La situación general de los pagos que se efectúan en los programas de afiliación está caracterizada, en general, por tener una estructura de comisiones débil y falta de comunicación entre las plataformas y sus clientes. La tendencia general, sin embargo, está cambiando este panorama y lleva a las plataformas a considerar a los afiliados como sus partners, lo que trae como consecuencia ofrecer un modelo de comisión atractivo y motivante y también, tener en cuenta las necesidades de los afiliados. Los pagos se están efectuando diferenciando a los soportes, así ofrecen pago por transferencia bancaria, paypal u otra modalidad. También, en el caso de que el afiliado, pese a residir en España quiera cobrar en otra moneda que no sea el euro, podría hacerlo. Del mismo modo los pagos tienden a ser efectivos nada más el cliente efectúa una compra ó realiza una acción.

Por otro lado, debemos investigar con la red qué modelos de comisión exitosos se están aplicando en la industria y averiguar qué comisiones están 
ofreciendo los competidores. A la hora de planificar a largo plazo, es también importante definir promociones especiales para, por ejemplo, diferentes fechas en el año, como Semana Santa, el Día de San Valentín o Navidad. Las promociones son otra manera de motivar a los afiliados, ya que son buenas oportunidades de incrementar los ingresos.

Nuevos Players y apertura al exterior: el Marketing de Resultados tiene cada vez más adeptos, pero estos resultados para que se mantengan y se incrementen en el tiempo, requieren una evolución en aspectos tan decisivos como es la adaptación de los players a los nuevos modelos. Se hace necesario que las plataformas agreguen a su cartera de clientes nuevos players, extranjeros, los Latam son muy adecuados por el idioma, del campo del gran consumo, para poder ofrecerles a los clientes productos que hasta este momento no se habían podido ofrecer y que provocaba que los compraran fuera. Además el cambio en los modelos de pricing debe ser evidente, con tendencia a modelos híbridos como en el resto de Europa.

Formatos creativos: de cara a presentar los productos de manera óptima y regular se debe ofrecer a los afiliados propuestas creativas, atractivas y actualizadas. No sólo ofrecer formatos de banner estándar, sino también links y formatos más originales, de manera que los afiliados puedan adaptarlos a sus páginas Web. También, es de gran ayuda si se sube información sobre el producto, como imágenes, precio o su descripción, a la base de datos para que se actualice automáticamente. Esto facilita que los afiliados creen propuestas creativas individuales y a medida para integrarlas en sus Webs. El Marketing de Afiliación no sólo cubre las páginas Web de información tradicional con banners, sino también una gran variedad de modelos de negocio para afiliados.

Incremento de colaboración. Las plataformas de afiliación, tal y como hemos analizado, infravaloraban la importancia de una colaboración y comunicación continua y personal con sus afiliados y anunciantes, aunque pronto reaccionaron y fueron conscientes de que era imprescindible para el éxito de los programas. La tendencia es a informar sobre todas las 
novedades y cambios, como posibles oportunidades adicionales para incrementar los ingresos, el crecimiento de la gama de productos, o los cambios que pueda haber en la estructura de comisiones. Mantener una continuidad con emails o newsletter es útil, pero mantener reuniones periódicas puede ser una buena manera de mejorar la relación. Todo ello significa que un afiliado y un anunciante motivado incrementarán las ventas de sus programas.

\section{CONCLUSIONES}

Dentro de este panorama podemos afirmar que el sector de Internet goza de una buena salud con datos que así lo avalan como el crecimiento en el número de internautas, el desarrollo del comercio electrónico o el aumento en el consumo del medio. Todos estos son argumentos más que de peso para convencer a una gran parte de anunciantes que todavía no creen en Internet.

En este sentido la fórmula de pago por resultados cobra más fuerza que nunca y debería ser una buena alternativa para aquellas empresas que quieren adentrarse en el mundo de Internet controlando mucho la inversión y midiendo exactamente el retorno. Existen sectores con un gran potencial de crecimiento en el Marketing de Afiliación como la moda, gran consumo o alimentación por citar sólo algunos ejemplos. Esas mismas empresas en países como Francia, Reino Unido o Alemania están dedicando presupuestos importantes en Performance Marketing y aprovechando todas las bondades del canal online para llegar a sus clientes. En todos los casos es muy importante que el anunciante se informe de lo que está haciendo su empresa y sus competidores en mercados más avanzados e intente poner en práctica muchas de las cosas que han funcionada en esos países en España.

Con todos estos ingredientes, tanto el sector de Internet en general como el sector de los anunciantes y afiliados, están ante una gran 
oportunidad y deben por ello, aprovechar las bondades del medio y rentabilizarlo, más en estos momentos de turbulencias económicas. 


\section{BIBLIOGRAFÍA}

Bruner, R.E. et al (2001), NetResults.2- Best Practices for Web Marketing. New Riders Publishing. Indianapolis.

Cerezo, J. (2009), "Ver más allá del banner”, en SANAGUSTíN, E. (Editor), Claves del Nuevo Marketing. Gestión 2000. Deusto

Jiménez, R. (2009), "Conocer Las Redes de Afiliados", en SANAGUSTÍN, E. (Editor), Claves del Nuevo Marketing. Gestión 2000. Deusto

IAB \& PwC (2011), Estudio sobre Inversión Publicitaria en Medios Digitales. Resultados del año 2010. (En línea). IAB Spain y Price Water House Coopers. Disponible en la Web www.iabspain.net. Se puede descargar en http://www.iabspain.net/descargas/descarga.php?id =155

Interactive Advertising Bureau (2010), Libro blanco del Marketing de Afiliación. (En línea). Cuadernos de comunicación Interactiva. Revista de Comunicación Interactiva y el Marketing Digital. Vol. 4. Ed. EDIPO S.A. Madrid. Disponible en la Web www.iabspain.net. Se puede descargar en http://www.iabspain.net/ver.php?id_categoria=9\&mod=descargas

Murillo, J (2008), "Cómo rentabilizar nuestro tráfico y nuestro contenido". Foro de Internet. Madrid. (Conferencia)

Rocco, E. y Dagnino, G. (2009), Coopetition Strategy: Theory, experiments and cases. . Ed. Taylor \& Francis. Estados Unidos.

SILVERSTEIN, B. (2001), "Developing Internet Partnerships". Direct Marketing. № 63(11), pp. 33-43.

Stokes, R (2010), Ultimate Guide to Pay-Per-Click Advertising. Ed McGraw-Hill. Nueva York. Estados Unidos 
Valdivielso, J.L. (2010), Retos del marketing de Resultados en 2010. OMExpo Madrid .

Valueclick. (2011), Informe Resultados Segundo Trimestre 2011. (En línea). Disponible en la Web http://ir.valueclick.com. Se puede descargar en http://ir.valueclick.com/releasedetail.cfm?ReleaseID=596192

Para citar este artículo:

Rodríguez Vázquez, Clide - Rodríguez Fernández, Magdalena (20-06-2012). LA INTELIGENCIA EN LA EMPRESA Y EL MARKETING DE AFILIACIÓN.

REDMARKA - CIECID - Unidad de Investigación en Marketing Aplicado-Universidad de A Coruña

Año V, Número 8, V2, pp.53-87

ISSN 1852-2300

URL del Documento : cienciared.com.ar/ra/doc.php? $\mathrm{n}=1665$

URL de la Revista : cienciared.com.ar/ra/revista.php?wid=39 